\title{
Laparoscopy versus open adrenalectomy in patients with solid tumor metastases: results of a multicenter European study
}

\author{
Pablo Moreno $^{1}$, Aitor de la Quintana Basarrate ${ }^{2}$, Thomas J. Musholt ${ }^{3}$, Ivan Paunovic ${ }^{4}$, Marco Puccini ${ }^{5}$, \\ Óscar Vidal ${ }^{6}$, Joaquín Ortega ${ }^{7}$, Jean-Louis Kraimps ${ }^{8}$; the European Study Group for Metastatic \\ Adrenalectomy*
}

${ }^{1}$ Unidad de Cirugía Endocrina, Hospital Universitari de Bellvitge, L'Hospitalet de Llobregat, Barcelona, Spain; ${ }^{2}$ Unidad de Cirugía Endocrina, Servicio de Cirugía General, Hospital de Cruces, Barakaldo, Bizkaia, Spain; ${ }^{3}$ Endocrine Surgery Section, Department of General, Visceral-, and Transplantation, University Medical Center of the Johannes Gutenberg University, Mainz, Germany; ${ }^{4}$ Center for Endocrine Surgery, Clinical Centre of Serbia Medical School, University of Belgrade, Belgrade, Serbia; ${ }^{5}$ Department of General Surgery, Cisanello Hospital, University of Pisa, Pisa, Italy; ${ }^{6}$ Department of General \& Endocrine Surgery, ICMDiM, Hospital Clinic-IDIBAPS, University of Barcelona, Barcelona, Spain; ${ }^{7}$ Department of Surgery, Hospital Clínico Universitario, University of Valencia, Valencia, Spain; ${ }^{8}$ Department of Endocrine Surgery, Jean Bernard Hospital, Poitiers University, Poitiers, France

Contributions: (I) Conception and design: P Morneo; (II) Administrative support: P Morneo; (III) Provision of study materials or patients: All Authors; (IV) Collection and assembly of data: P Morneo; (V) Data analysis and interpretation: P Morneo; (VI) Manuscript writing: All authors; (VII) Final approval of manuscript: All authors.

Correspondence to: Pablo Moreno, MD, PhD. Chief Endocrine Surgery Unit, Hospital Universitari de Bellvitge, Institute d'Investigació Biomédica de Bellvitge (IDIBELL), Feixa Llarga s/n, E-08907 L'Hospitalet de Llobregat, Barcelona, Spain. Email: 25108pml@gmail.com.

\begin{abstract}
Background: The outcome of adrenalectomy carried out by laparoscopy or open surgery for solid tumor metastases was assessed.

Methods: A total of 317 patients with histologically confirmed adrenal metastatic disease collected from 30 centres in Europe underwent adrenalectomy by laparoscopy $(n=146)$ or open laparotomy $(n=171)$. Differences between laparoscopic and open adrenalectomy were assessed by a single Cox analysis for both
\end{abstract}

\footnotetext{
* European Group for Metastatic Adrenalectomy (members by alphabetical order): Elisabeth Bollo Arocena, MD (Hospital Universitario Donostia, San Sebastián-Donostia, Spain); Mari Fe Candel Arenas, MD, PhD (Hospital General Universitario Reina Sofía, Universidad de Murcia, Murcia, Spain); Ignacio Escoresca, MD (Hospital Juan Ramón Jiménez, Huelva, Spain); Iñigo García Sanz, MD (Hospital Universitario de la Princesa, Universidad Autónoma de Madrid, Madrid, Spain); Óscar González López, MD, PhD (Hospital Universitari Vall d'Hebron, Universitat Autònoma de Barcelona, Barcelona, Spain); María-Teresa Gutiérrez Rodríguez, MD (Hospital Universitario de Basurto, Universidad del País Vasco, Bilbao, Bizkaia, Spain); Maurizio Iacobone, MD, PhD (University of Padua, Padua, Italy); Jean-Louis Kraimps, MD (Jean Bernard Hospital, Poitiers University, Poitiers, France); Kerstin Lorenz, MD (University of Halle-Wittenberg, Halle/ Saale, Germany); Jesús Martín, MD, PhD (Hospital General Universitario de Ciudad Real, Ciudad Real, Spain); Juan Manuel Martos, MD (Hospital Universitario Virgen del Rocío, Sevilla, Spain); Pablo Moreno, MD, PhD, FEBS (Hospital Universitari de Bellvitge, L'Hospitalet de Llobregat, Barcelona, Spain); Thomas J. Musholt, MD, FEBS (University Medical Center of the Johannes Gutenberg University, Mainz, Germany); Joaquín Ortega, MD, PhD (Hospital Clínico Universitario, University of Valencia, Valencia, Spain); Ivan Paunovic, MD, PhD (Clinical Centre of Serbia Medical School, University of Belgrade, Belgrade, Serbia); José M. del Pino, MD (Hospital Universitario Nuestra Señora de la Candelaria, Tenerife, Spain); Carlos D. del Pozo, MD, PhD (Hospital Dr. Peset, Valencia, Spain); Marco Puccini, MD (Cisanello Hospital, University of Pisa, Pisa, Italy); Aitor de la Quintana Basarrate, MD (Hospital de Cruces, Barakaldo, Bizkaia, Spain); José M. Ramia, MD, PhD (Hospital Universitario de Guadalajara, Guadalajara, Spain); José I. Rodríguez, MD (Hospital Universitari Josep Trueta, Girona, Spain); José M. Rodríguez, MD, PhD (Hospital Universitario Virgen de la Arrixaca, Murcia, Spain); David Scott-Coombes, MD, FRCS (University Hospital of Wales, Cardiff, Wales, UK); Ernesto Tobalina Aguirrezábal, MD (Hospital Galdakao-Usansolo, Galdakao, Bizkaia, Spain); Jaime Vázquez Echarri, MD, PhD (Hospital Universitario Severo Ochoa, Universidad Alfonso X Sabio, Leganés, Madrid, Spain); José Nuño Vázquez-Garza, MD (Hospital de Meixoeiro, Vigo, Spain); Enrique Veloso, MD, PhD (Hospital Mútua de Terrassa, Terrassa, Barcelona, Spain); Óscar Vidal, MD, PhD (ICMDiM, Hospital Clinic-IDIBAPS, University of Barcelona, Barcelona, Spain); and Jesús Villardel-Moral, MD, PhD (Hospital Universitario Virgen de las Nieves, Granada, Spain).
} 
procedures.

Results: The median overall survival was 24.0 [95\% confidence interval (CI): 21.4-26.6] months for open adrenalectomy and 45.0 (95\% CI: 22.6-67.4) for laparoscopic adrenalectomy $(\mathrm{P}=0.008)$. Survival rates were $68 \%, 49 \%, 35 \%$ and $29 \%$ at $1,2,3$ and 5 years for open surgery vs. $88 \%, 62 \%, 52 \%$ and $46 \%$ for laparoscopy, respectively. In the subgroup of R0 resections, the difference in survival in favor of laparoscopy (median 46 vs. 27 months) was marginally significant ( $\mathrm{P}=0.073)$. Renal cancer [hazard ratio (HR) 0.42; 95\% CI: $0.23-0.76, \mathrm{P}=0.005$ ], surgery of the primary tumor (HR 0.33; 95\% CI: 0.19-0.54), and use of chemotherapy (HR 0.62; 95\% CI: 0.43-0.88) were associated with a better survival, whereas type of resection (R1/R2 vs. R0) was associated with a worse prognosis (HR 2.29; 95\% CI: 1.52-3.44, P<0.001).

Conclusions: Laparoscopic adrenalectomy patients showed a longer survival than open adrenalectomy individuals, as minimally invasive approach was attempted more common in less advanced disease which led to higher number of R0 resections.

Keywords: Adrenalectomy; open adrenalectomy; laparoscopic adrenalectomy; survival; retrospective study

Submitted Sep 05, 2019. Accepted for publication Oct 15, 2019.

doi: $10.21037 /$ gs.2019.10.15

View this article at: http://dx.doi.org/10.21037/gs.2019.10.15

\section{Introduction}

Several studies have reported that adrenalectomy improves outcome in selected patients with metastatic disease, particularly in cases of isolated lesions and metachronous metastasis (1-6). In patients with isolated adrenal metastases, a more favourable survival for adrenal resection has been reported as compared with nonoperative approaches $(7,8)$. Adrenal metastasis is often confined within the gland itself, providing more chances to achieve en bloc removal. In the context of established indications for surgical extirpation of adrenal masses, laparoscopic adrenalectomy has become the gold standard for removal of most benign tumours and may be used for malignant lesions as well (9-12).

The laparoscopic approach has improved immediate surgical results as compared to the open procedure for the treatment of benign tumours, with the advantages of less blood loss, minimal invasion, quicker recovery, fewer complications, and shorter duration of hospitalization (13-16). However, there is much less experience regarding the use of laparoscopic adrenalectomy in metastatic adrenal disease. Adrenalectomy though the laparoscopic route as compared with open surgery achieves similar oncological results with less morbidity $(17,18)$, but homogeneous patient-based series comparing outcome differences between laparoscopic and conventional open adrenalectomy in patients metastatic adrenal disease are needed.

In a previous study of patients undergoing adrenalectomy for solid tumor metastasis included in a European multicenter survey, long-term survival in selected patients was shown after operative removal of adrenal metastasis (4). This retrospective study performed in the same population was designed to assess the outcome in oncological terms of removal of adrenal metastasis by laparoscopy or open surgery.

\section{Methods}

The design and characteristics of the study has been previously described (4). Briefly, this was a retrospective and multicenter survey with data provided by Units of Endocrine Surgery of 30 hospitals from UK, Italy, France, Germany, Serbia, and Spain. From 1999 to 2001, all consecutive patients with metastases to the adrenal gland in which adrenalectomy could be successfully performed, with histologically proven malignancy were included. Patients with primary renal tumors involving the adrenal glands and those in which the time elapsed between adrenalectomy to the last follow-up visit was not specified were excluded. This study aimed to assess the outcome of laparoscopic adrenalectomy $v s$. open adrenalectomy.

An ad hoc questionnaire was completed over 1-year period (July 2010-2011) with clinical information collected from medical records, including demographics, primary tumorrelated data, diagnostic techniques, side of the adrenal 
mass, extra-adrenal disease extension, type of surgical approach (laparoscopy vs. open laparotomy), completeness of resection (R0, complete resection, $\mathrm{R} 1$, microscopic margin involvement, R2, gross macroscopic tumor); isolated adrenalectomy (limited to the adrenal gland), extended adrenalectomy (resection of contiguous organs with lymphadenectomy), duration of surgical procedure, length of hospitalization, postoperative complications, adjuvant chemotherapy, size of the tumor, length of followup, and vital status at the last follow-up control. When the laparoscopic approach was converted to a laparotomy, these cases were considered as open.

In all cases, histopathologic reports were reviewed. Metastases detected at $\leq 6$ and $>6$ months after treatment of the primary tumor were classified as synchronous and metachronous, respectively. In patients treated with bilateral adrenalectomy in different procedures, the type of surgery and outcome of the first adrenalectomy was analyzed. Overall survival was defined as the time from adrenalectomy up to the date of the last follow-up or death.

\section{Statistical analysis}

Categorical variables are expressed as frequencies and percentages, and continuous variables as mean and standard deviation $( \pm \mathrm{SD})$. Follow-up time and survival time are expressed as median values and interquartile range (IQR) (25th-75th percentile). The Kaplan-Meier method was used to estimate survival, with median values and $95 \%$ confidence intervals (CIs) for 1, 2, 3, and 5 years survival rates. Survivals for the different surgical procedures (laparoscopy vs. open laparotomy) and for the subset of patients who underwent $\mathrm{R} 0$ resections were also estimated with the Kaplan-Meier method, with differences analyzed with the log-rank test. Bivariate Cox analysis was used to assess the relationship between each variable with survival. Data are expressed as hazard ratio (HR) and 95\% CI. Variables statistically significant or marginally significant in the bivariate analysis were analyzed in a Cox proportional hazards regression model with backward stepwise selection. Statistical significance was set at $\mathrm{P}<0.05$. Data were analyzed using the SPSS statistical program (version 18.0) (IMB Corp.).

\section{Results}

The study population included 317 patients, 171 (53.9\%) undergoing open adrenalectomy and 146 (46.1\%) laparoscopic adrenalectomy. Table 1 shows relevant characteristics of patients in the two study groups. Statistically significant differences in the laparoscopic adrenalectomy group as compared to the open adrenalectomy group included higher percentages non-small cell lung cancer (NSCLC) and colorectal cancer patients; chemotherapy treatment; use of computed tomography (CT) scan only or positron emission tomography (PET) for diagnosis; a lower percentage of bilateral adrenal metastases; higher percentages of isolated adrenalectomy, R0 resections; shorter duration of operation and hospitalization; smaller tumor size; and higher percentage of adjuvant-treated patients. The median (IQR) length of follow-up was 21 [8-36] months for open adrenalectomy and 20 [10-35] for laparoscopic adrenalectomy.

As shown in Table 2, in the bivariate analysis, renal cancer as the type of primary tumor and surgical treatment were significantly associated with a better survival, whereas the presence of synchronous adrenal metastasis, extra-adrenal disease, extended adrenalectomy, R1/R2 resections, duration of surgery, tumor size, length of hospital stay, and open surgery (or converted) were associated with poor survival. In the multivariate Cox proportional hazards regression model (Table 2), risk factors for better survival were renal cancer (HR 0.42; 95\% CI: 0.23-0.76, $\mathrm{P}=0.005$ ), surgery of the primary tumor (HR 0.33; 95\% CI: $0.19-0.54$ ), and use of chemotherapy (HR 0.62; 95\% CI: 0.43-0.88), whereas type of resection (R1/R2 vs. R0) was associated with a worse prognosis (HR 2.29; 95\% CI: 1.52-3.44, $\mathrm{P}<0.001$ ). Open adrenalectomy (or converted) almost reached statistical significance (HR 1.37; 95\% CI: 0.971-1.925, $\mathrm{P}=0.073$ ).

The median (IQR) survival for the laparoscopy group was 45.0 (22.6-67.4) months as compared with 24.0 (21.4-26.6) months for the open adrenalectomy group (log-rank test, $\mathrm{P}=0.008$ ) (Figure 1). In patients treated with laparoscopic adrenalectomy, the survival rates at $1,2,3$, and 5 years were $88.4 \%, 62.4 \%, 52.0 \%$, and $46.0 \%$, respectively. In patients treated with open adrenalectomy, the survival rates were $67.9 \%$ at 1 year, $49.0 \%$ at 2 years, $35.3 \%$ at 3 years, and $29.2 \%$ at 5 years.

The median survival analysis in the subset of patients undergoing R0 resections was 46 (95\% CI: 13.695-78.305) months for laparoscopy-treated patients and 27.0 (95\% CI: 18.063-35.937) for laparotomy-treated patients (log-rank test, $\mathrm{P}=0.073$ ).

\section{Discussion}

Results of the present study show a better survival for 
Table 1 Salient data of 317 patients with adrenal metastatic disease treated with open adrenalectomy and laparoscopic adrenalectomy

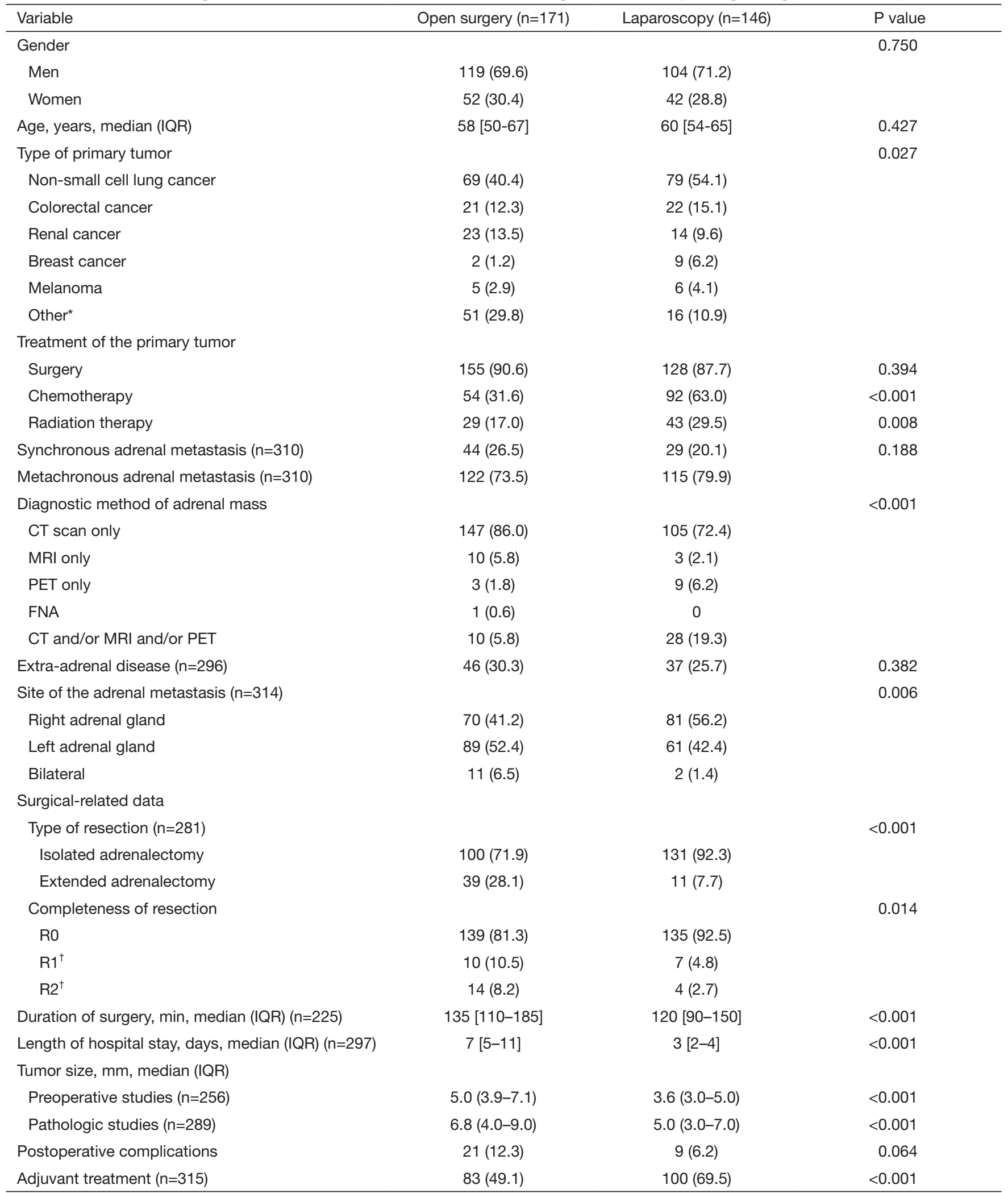

Data as frequencies and percentages in parenthesis unless otherwise stated. *, for the purpose of analysis breast cancer and melanoma are included in the "other" category; ${ }^{\dagger}, \mathrm{R} 1$ and R2 analyzed in a single category (R0 vs. R1 or R2). 
Table 2 Bivariate Cox analysis and multivariate Cox proportional hazard regression analysis

\begin{tabular}{|c|c|c|c|c|}
\hline Variables & \multicolumn{2}{|c|}{ Bivariate analysis } & \multicolumn{2}{|c|}{ Multivariate analysis } \\
\hline Age, years & $0.997(0.983-1.012)$ & 0.732 & & \\
\hline Gender (male vs. female) & $1.105(0.785-1.554)$ & 0.567 & & \\
\hline \multicolumn{5}{|l|}{ Type of primary tumor } \\
\hline Colorectal cancer & $0.847(0.513-1.399)$ & 0.516 & & \\
\hline Renal cancer & $0.494(0.279-0.873)$ & 0.015 & $0.420(0.231-0.764)$ & 0.005 \\
\hline Other & $1.222(0.861-1.735)$ & 0.262 & & \\
\hline \multicolumn{5}{|l|}{ Treatment of the primary tumor } \\
\hline Radiotherapy (yes vs. no) & $0.956(0.660-1.384)$ & 0.811 & & \\
\hline Synchronous adrenal metastasis vs. metachronous & $1.436(1.014-2.033)$ & 0.041 & & \\
\hline Extra-adrenal disease (presence vs. absence) & $1.460(1.030-2.070)$ & 0.033 & & \\
\hline \multicolumn{5}{|l|}{ Site of adrenal metastasis } \\
\hline Left adrenal gland (reference category) & 1 & & & \\
\hline Right adrenal gland & $0.957(0.701-1.306)$ & 0.781 & & \\
\hline Bilateral involvement & $0.813(0.329-2.007)$ & 0.653 & & \\
\hline Extended adrenalectomy (yes vs. isolated adrenalectomy) & $1.549(1.063-2.257)$ & 0.023 & & \\
\hline Length of hospital stay (days) ${ }^{\star}$ & $1.022(1.001-1.044)$ & 0.041 & & \\
\hline Postoperative complications (yes vs. no) & $1.272(0.788-2.055)$ & 0.325 & & \\
\hline Adjuvant treatment after adrenalectomy (yes vs. no) & $0.881(0.645-1.202)$ & 0.424 & & \\
\hline Open surgery (or converted) & $1.538(1.114-2.124)$ & 0.009 & $1.367(0.971-1.925)$ & 0.073 \\
\hline
\end{tabular}

Risk factors for survival in patients undergoing adrenalectomy for solid tumor metastasis. *, increased risk for each year of age, each minute of operative time, $1 \mathrm{~mm}$ of the tumor, and day of hospital stay.

patients with adrenal metastatic disease undergoing adrenalectomy via the laparoscopic route as compared to open surgery. Resection of adrenal metastasis through open surgery was a significantly associated with a poor survival in the bivariate analysis, although this variable was marginally significant in the multivariate Cox proportional hazards regression model.

Better outcomes were associated with laparoscopic adrenalectomy than those obtained after open operations. These results may be explained by different factors, including a reduced tumor size (both at preoperative and histopathological studies), fewer patients requiring extended 


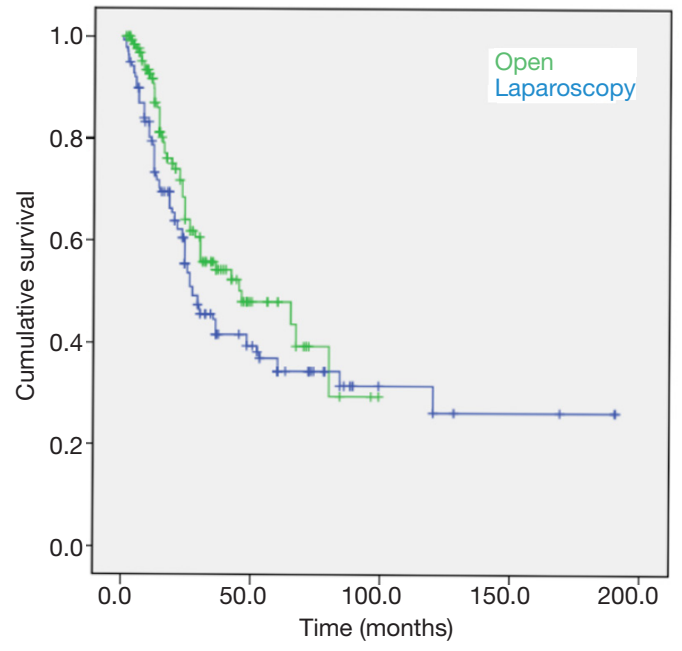

Figure 1 Differences in survival according to the surgical procedure (open surgery or laparoscopic adrenalectomy).

adrenalectomy, a higher number of primary tumors treated surgically and patients given chemotherapy for the primary malignant lesion, and a higher rate of complete resections (R0) in the laparoscopic group as compared to the open method. In relation to the effect of completeness of removal of adrenal metastasis, analysis of the subset of patients who underwent $\mathrm{R} 0$ resections, the length of surgical was longer for the laparoscopic approach (46 vs. 27 months) but the difference was marginally significant. The wellknown benefits of minimally invasive operations already reported by other authors (13-15), such as prompt recovery, a reduced rate of postoperative complications, and shorter length of hospitalization were also documented in our study.

Laparoscopic adrenalectomy for malignancy is still controversial. Although data from small series of patients have shown that laparoscopic radical adrenalectomy is feasible and can be performed with acceptable outcomes in the carefully selected patients with metastases (17,19-22), the present study presents a comparison of oncologic results in a large cohort of patients undergoing adrenalectomy for adrenal metastatic disease, $46 \%$ of which were operated on through the laparoscopic approach, using the transperitoneal route in most cases. Of note, strict homogeneous selection criteria and the long follow-up period are strengths of the study. However, because this was a retrospective, multicenter survey study, no specific hypothesis was pre-established Also, laparoscopic or open adrenalectomy was performed according to decision of the attending endocrine surgeon.
In relation variables associated with survival, renal cancer as the type of primary tumor and surgical treatment were significantly associated with a better survival, whereas survival was negatively affected by synchronous adrenal metastasis, extra-adrenal disease, extended adrenalectomy, R1/R2 resections, duration of surgery, tumor size, length of hospital stay, and open surgery (or converted). In the multivariate analysis, risk factors for better survival were renal cancer, surgery of the primary tumor, and use of chemotherapy, whereas type of resection (R1/R2 vs. R0) was associated with a worse prognosis.

On the other hand, the survival rates at 1, 2, 3, and 5 years after surgery were better for patients treated with laparoscopic adrenalectomy than for those undergoing open adrenalectomy. However, we found a favorable 5 -year survival rate of $46 \%$ of patients in the laparoscopy group and $29.2 \%$ of patients in the open surgery group, although our series included more than one third of patient with extra-adrenal disease at the time of adrenalectomy.

In summary, results of this retrospective multicenter review show that laparoscopic adrenalectomy was feasible for the treatment of selected patients with metastatic adrenal disease. Most importantly, this surgical approach was associated with a significantly longer survival as compared to open adrenalectomy, probably in relation to a higher number of $\mathrm{R} 0$ resections.

\section{Acknowledgments}

The authors thank Marta Pulido, MD, for editing the manuscript and editorial assistance.

\section{Footnote}

Conflicts of Interest: The authors have no conflicts of interest to declare.

Ethical Statement: The authors are accountable for all aspects of the work in ensuring that questions related to the accuracy or integrity of any part of the work are appropriately investigated and resolved.

\section{References}

1. Vazquez BJ, Richards ML, Lohse CM, et al. Adrenalectomy improves outcomes of selected patients with metastatic carcinoma. World J Surg 2012;36:1400-5.

2. Tanvetyanon T, Robinson LA, Schell MJ, et al. Outcomes 
of adrenalectomy for isolated synchronous versus metachronous adrenal metastases in non-small-cell lung cancer: a systematic review and pooled analysis. J Clin Oncol 2008;26:1142-7.

3. Collinson FJ, Lam TK, Bruijn WM, et al. Long-term survival and occasional regression of distant melanoma metastases after adrenal metastasectomy. Ann Surg Oncol 2008;15:1741-9.

4. Moreno P, de la Quintana Basarrate A, Musholt TJ, et al. Adrenalectomy for solid tumor metastases: results of a multicenter European study. Surgery 2013;154:1215-22; discussion 1222-3.

5. Howell GM, Carty SE, Armstrong MJ, et al. Outcome and prognostic factors after adrenalectomy for patients with distant adrenal metastasis. Ann Surg Oncol 2013;20:3491-6.

6. Bastian S, Clerici T, Neuweiler J, et al. Surgical resection of isolated adrenal metastases in patients with non-small cell lung cancer: a single-institution experience and review of the literature. Onkologie 2011;34:665-70.

7. Sarela AI, Murphy I, Coit DG, et al. Metastasis to the adrenal gland: the emerging role of laparoscopic surgery. Ann Surg Oncol 2003;10:1191-6.

8. Luketich JD, Burt ME. Does resection of adrenal metastases from non-small cell lung cancer improve survival? Ann Thorac Surg 1996;62:1614-6.

9. Micali S, Peluso G, De Stefani S, et al. Laparoscopic adrenal surgery: new frontiers. J Endourol 2005;19:272-8.

10. Hall DW, Raman JD. Has laparoscopy impacted the indications for adrenalectomy? Curr Urol Rep 2010;11:132-7.

11. Miccoli P, Raffaelli M, Berti P, et al. Adrenal surgery before and after the introduction of laparoscopic adrenalectomy. Br J Surg 2002;89:779-82.

Cite this article as: Moreno P, de la Quintana Basarrate A, Musholt TJ, Paunovic I, Puccini M, Vidal Ó, Ortega J, Kraimps JL; the European Study Group for Metastatic Adrenalectomy. Laparoscopy versus open adrenalectomy in patients with solid tumor metastases: results of a multicenter European study. Gland Surg 2020;9(Suppl 2):S159-S165. doi: $10.21037 /$ gs.2019.10.15
12. Kazaryan AM, Marangos IP, Rosseland AR, et al. Laparoscopic adrenalectomy: Norwegian single-center experience of 242 procedures. J Laparoendosc Adv Surg Tech A 2009;19:181-9.

13. Plaggemars HJ, Targarona EM, van Couwelaar G, et al. What has changed in adrenalectomy? From open surgery to laparoscopy? Cir Esp 2005;77:132-8.

14. Hazzan D, Shiloni E, Golijanin D, et al. Laparoscopic vs open adrenalectomy for benign adrenal neoplasm. Surg Endosc 2001;15:1356-8.

15. Brunt LM, Doherty GM, Norton JA, et al. Laparoscopic adrenalectomy compared to open adrenalectomy for benign adrenal neoplasms. J Am Coll Surg 1996;183:1-10.

16. Chuan-Yu S, Yat-faat H, Wei-hong D, et al. Laparoscopic adrenalectomy for adrenal tumors. Int J Endocrinol 2014;2014:241854.

17. Strong VE, D'Angelica M, Tang L, et al. Laparoscopic adrenalectomy for isolated adrenal metastasis. Ann Surg Oncol 2007;14:3392-400.

18. Silvio Estaba L, Madrazo González Z, Pujol Gebelli J, et al. Laparoscopic adrenalectomy for suspected isolated adrenal metastasis. Cir Esp 2007;81:197-201.

19. Moinzadeh A, Gill IS. Laparoscopic radical adrenalectomy for malignancy in 31 patients. J Urol 2005;173:519-25.

20. McCauley LR, Nguyen MM. Laparoscopic radical adrenalectomy for cancer: long-term outcomes. Curr Opin Urol 2008;18:134-8.

21. Adler JT, Mack E, Chen H. Equal oncologic results for laparoscopic and open resection of adrenal metastases. J Surg Res 2007;140:159-64.

22. Marangos IP, Kazaryan AM, Rosseland AR, et al. Should we use laparoscopic adrenalectomy for metastases? Scandinavian multicenter study. J Surg Oncol 2009; 100:43-7. 